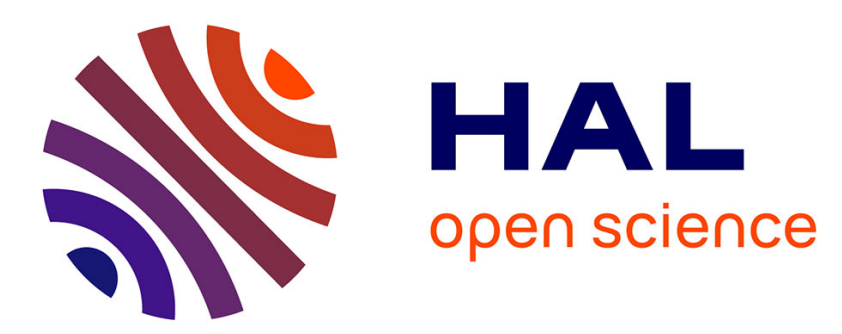

\title{
Molecular dynamics simulations as a way to investigate the local physics of contact mechanics: a comparison between experimental data and numerical results
} M Solar, H Meyer, C Gauthier, O Benzerara, H Pelletier, R Schirrer, J Baschnagel

\section{To cite this version:}

M Solar, H Meyer, C Gauthier, O Benzerara, H Pelletier, et al.. Molecular dynamics simulations as a way to investigate the local physics of contact mechanics: a comparison between experimental data and numerical results. Journal of Physics D: Applied Physics, 2010, 43 (45), pp.455406. 10.1088/0022$3727 / 43 / 45 / 455406$. hal-00569743

\section{HAL Id: hal-00569743 \\ https://hal.science/hal-00569743}

Submitted on 25 Feb 2011

HAL is a multi-disciplinary open access archive for the deposit and dissemination of scientific research documents, whether they are published or not. The documents may come from teaching and research institutions in France or abroad, or from public or private research centers.
L'archive ouverte pluridisciplinaire HAL, est destinée au dépôt et à la diffusion de documents scientifiques de niveau recherche, publiés ou non, émanant des établissements d'enseignement et de recherche français ou étrangers, des laboratoires publics ou privés. 


\title{
Molecular dynamics simulations as a way to investigate the local physics of contact mechanics: a comparison between experimental data and numerical results
}

\author{
M. SOLAR, H. MEYER, C. GAUTHIER, O. BENZERARA \\ H. PELLETIER, R. SCHIRRER and J. BASCHNAGEL \\ Institut Charles Sadron UPR 0022, Campus CNRS de Cronenbourg, 23 rue du Loess \\ BP 84047, 67034 Strasbourg Cedex 2 (FR.) \\ E-mail: christian.gauthier@ics-cnrs.unistra.fr, \\ hendrik.meyer@ics-cnrs.unistra.fr, mathieu.solar@ics-cnrs.unistra.fr
}

\begin{abstract}
.
In this work, a mechanical analysis of normal contact using molecular dynamics (MD) simulations is presented. Conical indentation on amorphous polymer surfaces was simulated at various temperatures and indentation rates under displacement or load control. The results are qualitatively compared with experimental data from tests on epoxy materials with different glass transition temperatures $\left(T_{g}\right)$, and show good agreement with experiments. Moreover, MD simulations of nano-indentation tests allow us to estimate the mechanical properties of the polymer films studied as in experimental nano-indentation tests, which demonstrates the relevance of this approach.
\end{abstract}

PACS numbers: 62.25.-g, 61.41.+e, 62.20.Qp, 68.60.Bs, 81.40.Lm, 81.70.Bt

Submitted to: J. Phys. D: Appl. Phys.

\section{Introduction}

Contact with a surface probe is a mechanical test which allows one to investigate materials on the submicron scale, thanks to recent progress in instrumentation technology. In this way, one distinguishes $(i)$ "in-situ" testing where the contact is observed at all time points and (ii) "structure" testing, like for example classical depthsensing nano-indentation tests or indentation tests with an AFM tip, which allows one to estimate the contact stiffness for a submicron depth. The first type of test is not the subject of this work, see for example $[1,2,3]$. In the second type, the evaluation of a material property requires modeling of the tip/surface contact and depends on the richness of this modeling, see for example $[4,5]$. One may deduce from these tests that 
the contact between a rigid indenter and a polymer surface is governed by bulk properties (Young's modulus $E$, yield stress $\sigma_{y}$, bulk glass transition, etc ...) and surface properties (surface tension $\gamma$, surface energy, surface glass transition, etc ...). These properties can nevertheless be difficult to model or observe experimentally. In addition, the direction in which the tip is moving (normal contact = indentation; tangential contact $=$ scratching) gives the interfacial shear stress more or less importance.

\subsection{Problem and background}

Scratch resistance is an important mechanical property for the surfaces of bulk materials and for thin layer materials (e.g. glasses, varnishes ...). For polymer surfaces, this resistance can be improved by decreasing the local friction, minimizing the plastic strain or obtaining better recovery after scratching. Moreover, it was proven to be relevant to differentiate between the surface behavior and that of the bulk, by investigating the domain confined under the contact. This domain is at the interface between indenter and substrate (see for example $[6,7,8,9]$ ).

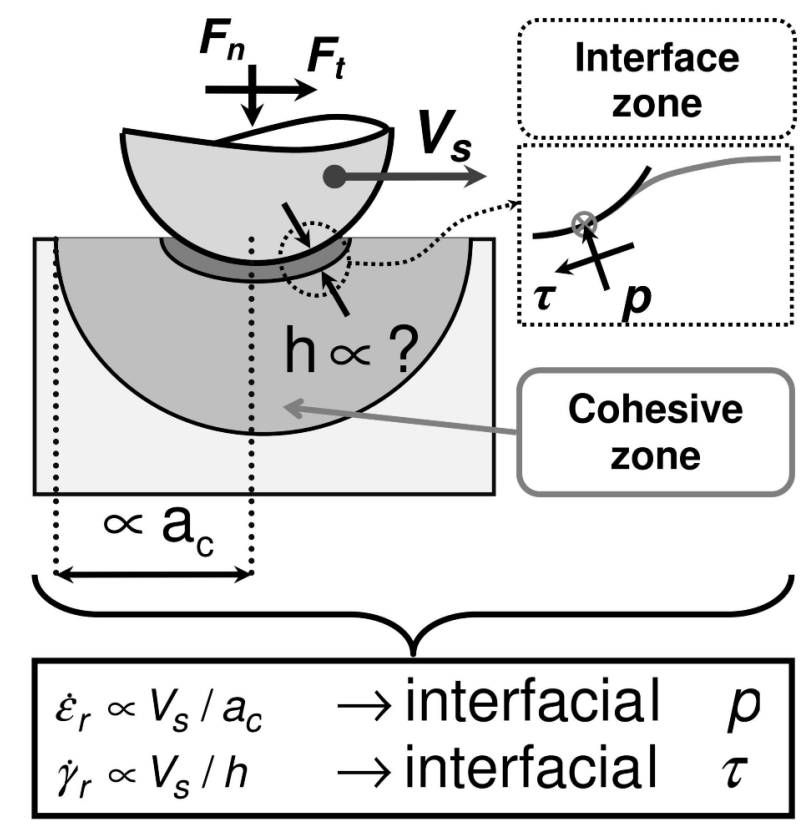

Figure 1. During scratching at velocity $V_{s}$, the representative strain rate $\dot{\epsilon}_{r} \propto V_{s} / a_{c}$ is thought to control the interfacial contact pressure $p$, while the representative shear strain rate $\dot{\gamma}_{r} \propto V_{s} / h$ is thought to control the interfacial shear stress $\tau$.

In the case of a micro/nano-scratch, the contact pressure and interfacial shear stress control the penetration of the tip into the material. It has been suggested by Briscoe [7] and more recently by Charrault et al. [6] that the representative strain rate of the contact (proportional to $V_{s} / a_{c}$, where $V_{s}$ is the scratching velocity and $a_{c}$ the contact 
radius) controls the mean contact pressure, whereas the interfacial shear stress (related to the local friction) is controlled by a representative shear strain rate of the contact (proportional to $V_{s} / h$, where $h$ is the unknown thickness of a small sheared layer under the contact, see figure 1). Consequently, the contact radius $a_{c}$ would seem to be the characteristic length for the bulk response, whereas the thickness $h$ of a small sheared layer would seem to be the characteristic length for the surface response of the material. It is actually assumed that $h \ll a_{c}[7]$ and an approximation can be found in the work of Charrault et al. [6]. A more complete study of the problem is however necessary. Thus, polymers present viscoelastic and viscoplastic properties and display complex behavior in the presence of confined shearing (friction, i.e. interfacial shear stress). The improvement of their surface behavior requires a better understanding of the local physics of their contact mechanics, during indentation and scratching.

\subsection{Why use molecular dynamics simulations?}

Continuum mechanics (CM) has enabled a better identification and comprehension of the mechanical stresses and strains to which a material is subjected during micro- or nano-indentation tests. Finite element (FE) simulations are commonly used to predict the mechanical behavior. However, the results depend on phenomenological models, which in turn rely on experimental observations and practical inverse analysis of the bulk behavioral laws. In addition the models use a macroscopic thermodynamic formulation [10]. The experimental analysis and practical inverse analysis are valid so long as the surface behavior is negligible in comparison with the bulk behavior. Surface tension will not influence the contact stiffness in such cases. On the other hand, the material is seen as a continuous medium in CM, thereby disregarding its molecular structure, so as to be able to use differential formalism. This approach is limited when the local physics contributes to the global behavior. Some phenomena (e.g. the local gradient of a mechanical property) cannot be predicted with CM, when the constitutive model contains no explicit law to describe such phenomena. The modeling of nano-indentation and nano-scratch tests lies at the limits of continuum mechanics since on the nano scale the behavior is a blend of the bulk mechanical behavior (described by phenomenological laws with a macroscopic thermodynamic formulation) and the local physics including surface properties (e.g. surface tension, local friction, adhesion, etc ...). Moreover, these surface properties are difficult to model or observe experimentally. The molecular dynamics (MD) simulation approach is more relevant in such situations because it considers molecular details and has a formulation based on statistical thermodynamics. As an example, MD simulations can predict changes in the behavior of a material from variations in thermodynamic parameters (e.g. pressure, temperature, volume, etc ...). Such simulations can be used to study local structural changes during a deformation process as in $[11,12,13,14,15]$. The simulated time and length scales are still small in comparison with CM (see figure 2). On the negative side, MD simulations currently require a lot of CPU time. Several groups have applied MD simulations to tribological 
problems on hard or crystalline surfaces, examining single asperities and lubricants $[16,17,18,19]$. There exist however very few attempts to examine nano-indentation on polymer surfaces (see for example $[20,21,22,23,24,25]$ ). This work presents a mechanical analysis of normal contact (indentation test) on amorphous polymer surfaces using MD simulations.

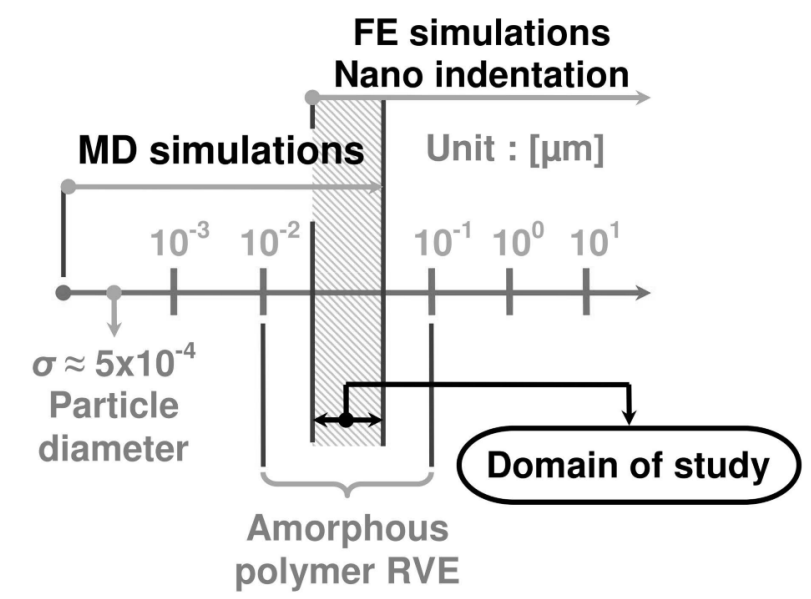

Figure 2. Relevant scales for $\mathrm{MD}$ and FE simulations of nano-indentation. This work was performed in the domain accessible to both techniques.

\section{Modeling the contact using molecular dynamics simulations}

MD simulations can predict particle motion inside matter by numerical integration of the classical equations of motion. Two independent programs implementing the Velocity Verlet algorithm were used: an in-house MD CODE and the LAMMPS (c) program [26]. A comparison of the two methods revealed no discrepancy for the present study. A detailed description of the principles of MD simulations may be found in $[27,28]$.

\subsection{Molecular dynamics simulations: some generalities}

As nano-indentation testing progressively approaches the representative volume element (RVE) of amorphous polymers, MD simulations become increasingly relevant. In the present work, the polymer was modeled by a generic "coarse-grained" model. A monomer refers to one or more group(s) of atoms belonging to a polymer chain and is regarded as a quasi-impenetrable sphere of diameter $\sigma$ and mass $m$. Indentation with a conical tip was simulated on films of linear amorphous polymer chains. Cross-linked polymers were excluded, since our linear polymer model only allows physical knots. It is also important to note that the results are presented in dimensionless Lennard-Jones $(\mathrm{LJ})$ units (superscript $*$ ). Table 1 lists the principal LJ units for the example of PMMA 
$\left(m=1.660 \cdot 10^{-25}[\mathrm{~kg}]\right.$, so $\left.M=100\left[\mathrm{~g} \cdot \mathrm{mol}^{-1}\right]\right)$ with reference to the work of Schnell et al. [29]. The superscript $*$ is omitted, except if confusion might occur.

Table 1. Comparison of the dimensionless Lennard-Jones units (LJ units, used in MD simulations) with SI units $\left(k_{B}\right.$ is the Boltzmann constant: $k_{B} \approx 1.380 \cdot 10^{-23}\left[J \cdot K^{-1}\right]$ and $\tau$ is the time unit: $\left.\tau=\sqrt{\left(m \cdot \sigma^{2}\right) / \epsilon}\right)$. Parameter $\sigma$ is the monomer diameter. Parameter $\epsilon$ is the minimum LJ potential.

\begin{tabular}{llll}
\hline LJ symbol & Designation & SI symbol & Order of magnitude \\
\hline$r^{*}=(1 / \sigma) \cdot r$ & length & $r[\mathrm{~m}]$ & $\sigma \approx 0.5 \cdot 10^{-09}[\mathrm{~m}]$ \\
$T^{*}=\left(k_{B} / \epsilon\right) \cdot T$ & temperature & $T[\mathrm{~K}]$ & $\epsilon / k_{B} \approx 8.5 \cdot 10^{+02}[\mathrm{~K}]$ \\
$E^{*}=(1 / \epsilon) \cdot E$ & energy & $E[\mathrm{~J}]$ & $\epsilon \approx 1.2 \cdot 10^{-20}[\mathrm{~J}]$ \\
$P_{h}^{*}=\left(\sigma^{3} / \epsilon\right) \cdot P_{h}$ & pressure & $P_{h}[\mathrm{~Pa}]$ & $\epsilon / \sigma^{3} \approx 9.4 \cdot 10^{+07}[\mathrm{~Pa}]$ \\
$t^{*}=(1 / \tau) \cdot t$ & time & $t[\mathrm{~s}]$ & $\tau \approx 1.9 \cdot 10^{+00}[\mathrm{ps}]$ \\
$F^{*}=(\sigma / \epsilon) \cdot F$ & load & $F[N]$ & $\epsilon / \sigma \approx 2.3 \cdot 10^{+01}[\mathrm{pN}]$ \\
$\rho^{*}=\left(\sigma^{3} / \mathrm{m}\right) \cdot \rho$ & mass per volume & $\rho\left[\mathrm{kg} \cdot \mathrm{m}^{-3}\right]$ & $\mathrm{m} / \sigma^{3} \approx 1.3 \cdot 10^{+03}\left[\mathrm{~kg} \cdot \mathrm{m}^{-3}\right]$ \\
$\gamma^{*}=\left(\sigma^{2} / \epsilon\right) \cdot \gamma$ & surface tension & $\gamma\left[\mathrm{N} \cdot \mathrm{m}^{-1}\right]$ & $\epsilon / \sigma^{2} \approx 4.4 \cdot 10^{-02}\left[\mathrm{~N} \cdot \mathrm{m}^{-1}\right]$ \\
\hline
\end{tabular}

\subsection{Modeling of indentation on polymer films}

The simulated polymer films (see figure 3) consisted of $N_{c}=192$ chains of $N_{p}=64$ monomers (thin polymer film) and $N_{c}=1536$ chains of $N_{p}=64$ particles (thick polymer film). For the $64 \times 192$ polymer film (resp. $64 \times 1536$ film), the dimensions of the simulation box were set to $30 \sigma, 30 \sigma$ and $30 \sigma$ (resp. $60 \sigma, 60 \sigma$ and $60 \sigma$ ). The dimensions of the films were the same as these of the simulation box in the $X$-and $Z$-directions. In the $Y$-direction, the first film (resp. the second) was $\approx 14-15 \sigma$ (resp. $\approx 27-28 \sigma$ ), the second film being twice as thick as the first. Periodic boundary conditions were applied only in the $X$ - and $Z$-directions of the simulation box. It is important to note that our chain length was close to the entanglement length of bead-spring melts (denoted $N_{e}$ ), which defines slightly entangled chains. This was calculated for example by Everaers et al. to be $N_{e} \approx 65[30]$ ( $N_{e}$ refers to the number of monomers between entanglements and is given by the number of monomers per Kuhn segment of the primitive path). In our polymer films entanglements had only a weak influence on the mechanical properties.

2.2.1. Polymer film model The equations of motion were integrated using the Velocity Verlet algorithm implemented in the MD CODE and LAMMPS (c) programs. The timestep in the first case was $0.01 \tau$ and $0.005 \tau$ in the second. For the indentations reported here, no significant influence of the timestep was observed. In scratch simulations, higher forces would be expected to occur and a smaller timestep would be necessary. Simulations were performed in the canonical ensemble, i.e. in the NVT system: $N=N_{c} N_{p}$, constant number of particles in the box; $V$, constant volume of the box; $T$, mean constant temperature of the box, where the film dilates with $T$. For the $64 \times 192$ polymer film, a DPD (dissipative particles dynamics) thermostat was used 
for $T$-regulation with a friction parameter $\zeta=0.5 \tau^{-1}$ [31]. For the $64 \times 1536$ polymer film, an NH (Nose-Hoover) thermostat was used with the parameters $\tau_{\text {damp }}=1.0 \tau$ and $\tau_{\text {drag }}=0.02$ (see LAMMPS (C) [32]. The two thermostats were checked and no significant differences were found concerning the global response of the polymer films. A flexible chain model was employed as a generic bead-spring model for linear polymer chains and a harmonic potential $U_{\text {bond }}$ imposed chain connectivity:

$$
U_{\text {bond }}\left(R_{i}\right)=0.5 \cdot k_{\text {bond }} \cdot\left(R_{i}-r_{0}\right)^{2},
$$

with a bond stiffness $k_{\text {bond }}=1111 \epsilon / \sigma^{2}$, an equilibrium bond length $r_{0}=0.967 \sigma$ and $R_{i}$ the bond length between consecutive monomers in the chain. This potential was shown to be very similar to that of the Bennemann model [33] (which uses a FENE potential) concerning the static and dynamic properties of the polymer film [34]. Thus, particles of the same chain which are not directly connected and particles of different chains interact through a truncated $\left(r_{c}=2.3 \sigma\right.$ is the cut-off radius) and shifted $(C=0.02684 \sigma) 12-6$ $L J$ potential $U_{L J}(2)$, where $r$ is the distance between two particles:

$$
U_{L J}(r)= \begin{cases}4 \epsilon\left[(\sigma / r)^{12}-(\sigma / r)^{6}\right]+C & \text { if } r<r_{c}, \\ 0 & \text { if } r \geq r_{c} .\end{cases}
$$

The parameter $C$ ensures potential continuity because forces on particles are numerically computed from the gradient operator of interaction potentials. In both cases $(64 \times 192$ and $64 \times 1536$ polymer films), the film was supported by a perfectly rigid wall and had a free surface (hydrostatic pressure $P_{h}=0$ at the surface). To model the monomer-wall interaction, a $12-6 L J$ potential $U_{\text {wall }}=U_{L J}(y)(2)$ was used, where $y$ denotes the distance of a particle from the wall in the $Y$-direction. A complete characterization of a similar polymer film model has been given by Peter et al. [34], and the same group has carried out a previous study of polymer films with a chain length of 64 monomers et al. [35]. In the case of the $64 \times 1536$ polymer film, the particles at the bottom of the simulation box (belonging to a volume of dimensions $L_{x}, 1.5 \sigma$ and $L_{z}$ in the $X-, Y$ and $Z$-directions, respectively) had a spring attachment to tether them to their initial position. Thus, a particle experienced a back force $\vec{F}_{\text {spring } \rightarrow i}=k_{\text {spring }}^{\text {attach }} \cdot\left(\vec{r}_{i}-\vec{r}_{\text {init }}\right)^{2}$ at each timestep, $\vec{r}_{i}$ being the position vector of particle $i$ at time $t$ and $\vec{r}_{\text {init }}$ its position vector at the start of the simulation. The stiffness of the attachment was fixed at $k_{\text {spring }}^{\text {attach }}=10 \epsilon / \sigma^{2}$ to avoid sliding of the film, for example during a scratch test. This condition was imposed on the monomers after the polymer film had been equilibrated at constant temperature without it, and before any mechanical test. The attachment had no influence on the indentation test as compared to the same test without. In our simulations, we took $\sigma=\epsilon=1$.

2.2.2. Indenter modeling and contact conditions For indentation tests on the $64 \times 192$ film (resp. $64 \times 1536$ film), the indenter was a smooth conical zone (resp. made of particles having the same diameter as those of the polymer film). The impenetrability 
condition, i.e. interaction between the tip and the polymer, was ensured by a short range repulsive LJ potential (2), $r$ becoming the normal distance between the cone surface and a film particle (resp. the distance between two particles). The values of $r_{c}$ and $C$ were chosen $\left(r_{c}=1.12 \sigma\right.$ and $\left.C=0.99982 \sigma\right)$ to ensure a purely repulsive interaction (frictionless contact for a perfectly smooth tip). The half-apex angle was $\theta$ and the tip was considered to be almost smooth. Concerning the vertical movement of the tip, a linear depth function $(d \delta / d t$ is constant, when the depth under the surface $\delta$ is controlled) and a linear load function $(d P / d t$ is constant, when the load on the tip $P$ is controlled) were employed. In the second case, a friction coefficient $\zeta_{i / f}=\left(P-F_{\text {polymer }}\right) / V_{\text {ind }}=100$ between the tip and the film was introduced to avoid numerical oscillation [20]. This was distinct from the local friction coefficient $\mu$. $P$ was the external load applied to the tip, $F_{\text {polymer }}$ the reaction force of the film acting on the tip and $V_{\text {ind }}=V$ the tip velocity in the $Y$-direction.

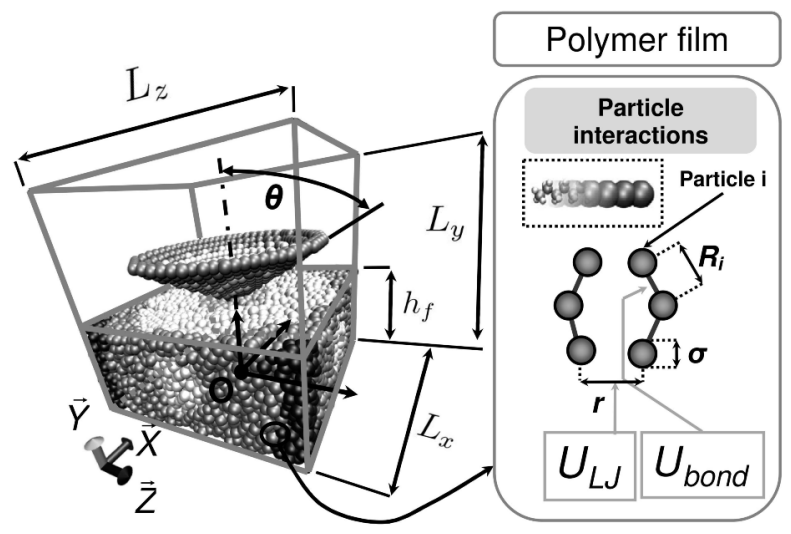

Figure 3. Coarse-grained model for $64 \times 192$ and $64 \times 1536$ films of a linear polymer. The tip is perfectly smooth $(64 \times 192$ polymer film) or modeled with particles $(64 \times 1536$ polymer film).

\subsection{Glass transition, film thickness and film height}

The polymer film dilates with increasing temperature, due to thermal expansion. To determine the mean glass transition $T_{g}$ of the film, the film thickness $h_{t h, f}$ was monitored during cooling, and $T_{g}$ was identified as the temperature where the slope of $h_{t h, f}$ changed. Such a pseudothermodynamic approach is commonly employed in experiments, and has been described for MD simulations by Peter et al. [34]. The film thickness $h_{t h, f}$ may be derived from the monomer density profile and is obtained by the Gibbs dividing surface (GDS) method. In our case, the mean glass transition $T_{g}$ of the $64 \times 192$ film (resp. $64 \times 1536$ film) was calculated to be $T_{g}=0.39 \pm 0.02$ (resp. $0.41 \pm 0.06$ ). Thus, the glass transition of the two polymer films was $\approx 0.40$. The film thickness $h_{t h, f}$ gives the average thickness, where the particles of the film are concentrated. It is not the 
height where the tip begins to come into contact with the film. Hence the mean film height $h_{f}$ was introduced, which is the local height below the tip. It can be derived from the monomer density profile and is obtained in the same way as $h_{t h, f}$ but without requiring the GDS method. A negligible load $P$ of 0 to 10 corresponds to this height. The mean film height $h_{f}$ is more appropriate to estimate the height where the tip comes into contact with the film (nano-indentation test). For the $64 \times 192$ (resp. $64 \times 1536$ ) polymer film, the film height $h_{f}$ was $14.04 \sigma$ (resp. $27.55 \sigma$ ) at $T=0.2$, and $14.50 \sigma$ (resp. $28.00 \sigma)$ at $T=0.4$. A difference between $h_{t h, f}$ and $h_{f}$ of about $10 \%$ could be observed.

\subsection{Nano-indentation tests: (P vs. $\delta)$ curves}

To avoid the influence of boundary effects on the results, one should apply the rules given by Bucaille et al. [36]. These rules concern the dimensions of the domain (i.e. the polymer film) and specify a width of 20 times the contact radius $a_{c}$, and a height of 6 times the penetration depth $\delta$.

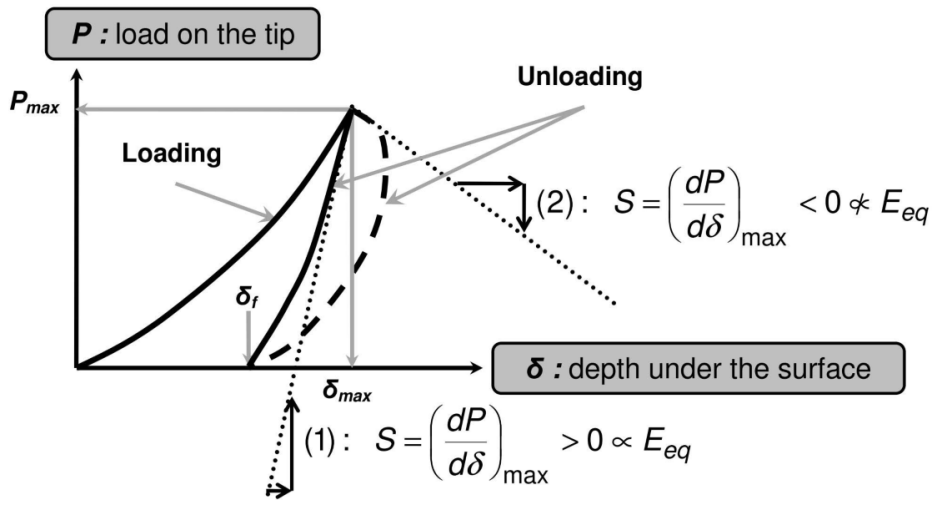

Figure 4. The $(P$ vs. $\delta)$ curve is important in nano-indentation tests. In the case of a semi-infinite medium, the initial slope of the unloading curve $(S)$ is proportional to the equivalent contact stiffness $\left(E_{\text {eq }}\right)$ for materials with elastoplastic behavior (case (1) in the figure) [4]. For materials with viscoelastic and/or viscoplastic behavior, creep can occur during the unloading and $S$ may be negative (case (2) in the figure) and is not proportional to $E_{e q}$. In the case of a finite medium, the substrate has an influence: $S$ is not proportional to $E_{e q}$.

Introducing the vertical confinement $\kappa=\delta / h_{f}$, a depth of $\delta \approx 5-10[\sigma]$ gives $\kappa \in[0.18 ; 0.37]$. The penetration was thus chosen to reach a significant depth (or some particle diameters), which was more than $\kappa=1 / 6 \approx 0.17$ allowed by the rules of Bucaille et al. [36]. Consequently, an influence of the perfectly rigid wall on the initial 
slope $S$ of the unloading curve ( $P$ vs. $\delta$ ) could be expected, more so for the $64 \times 192$ polymer film than for the $64 \times 1536$ film. Moreover, the contact diameter $2 a_{c}$ for $\delta=5[\sigma]$ and a conical tip with $\theta=70.3^{\circ}$ would be $27.9[\sigma]$, which is of the order of the box size. The lateral confinement (image of the tip due to periodic boundary conditions) should also have an influence. To take into account these observations and the size limits of the simulation box, conical tips with $\theta=60^{\circ}, \theta=45^{\circ}$ and $\theta=30^{\circ}$ were chosen for the $64 \times 192$ polymer film $\ddagger$ and a conical tip with $\theta=70.3^{\circ}$ for the $64 \times 1536$ polymer film in our numerical simulations. Indentation was simulated at $T=0.2\left(<T_{g}\right.$, in the glassy state) and $T=0.4\left(\approx T_{g}\right.$, in the rubbery state). The ( $P$ vs. $\left.\delta\right)$ curves are presented in figure 4: on the $X$-axis is the depth under the surface in $\sigma$ units (LJ units; the depth $\delta^{*}$ is denoted $\delta$ as remarked before and is some particle diameters in magnitude) and on the $Y$-axis the load on the tip in $\epsilon / \sigma$ units (LJ units; the load $P^{*}$ is denoted $P$ ).

2.4.1. Study of the loading To assist comprehension, a regression of the loading curve using the function $P=A \delta^{2}+C \delta$ is juxtaposed to the curve $(P$ vs $\delta$ ) during the loading phase. The parameter $A$ (resp. $C$ ) has the units of pressure (resp. stiffness) and quantifies the hardness of the polymer film.

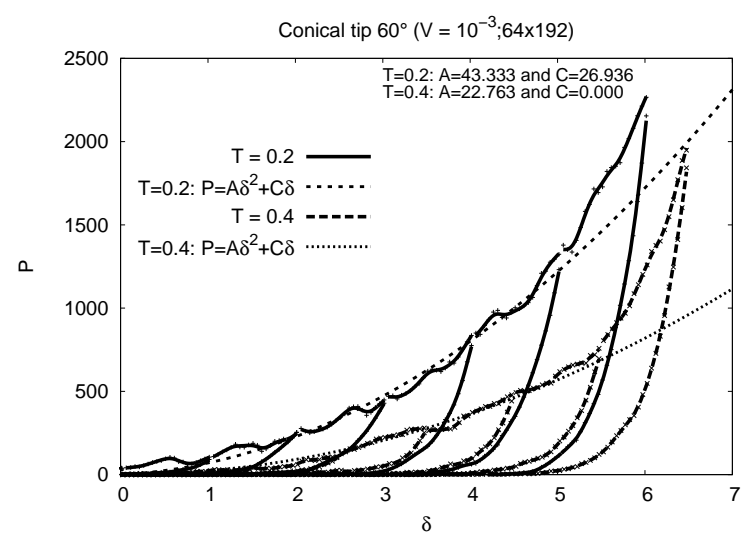

Figure 5. MD simulations of an indentation test with a linear $\delta$ function $(V=d \delta / d t=$ $10^{-3}$ ) at two different temperatures. The regressions with their appropriate functions are shown for $\delta \in[0 ; 5]$.

Figure 5 presents the results for a test with a linear displacement $(\delta)$ loading function (several indentations at several depths). This figure shows that a temperature rise leads to a decrease in the hardness of the polymer film at constant loading speed $(V$ is constant). The parameter $A$ passes from 43.33 at $T=0.2$ to 22.76 at $T=0.4$, for a fixed constant loading speed of $V=10^{-3}$. This may be explained by the fact that the thermal agitation of particles is higher at $T=0.4$ than at $T=0.2$. The higher the $\ddagger$ Numerical oscillations were observed in the last two cases. 
temperature, the faster the relaxation of the polymer chains, because the higher kinetic energy of the particles permits more frequent jumps from one LJ potential pit to an other. Due to this faster relaxation of the polymer chains, a smaller load on the tip $P$ is required to deform them.

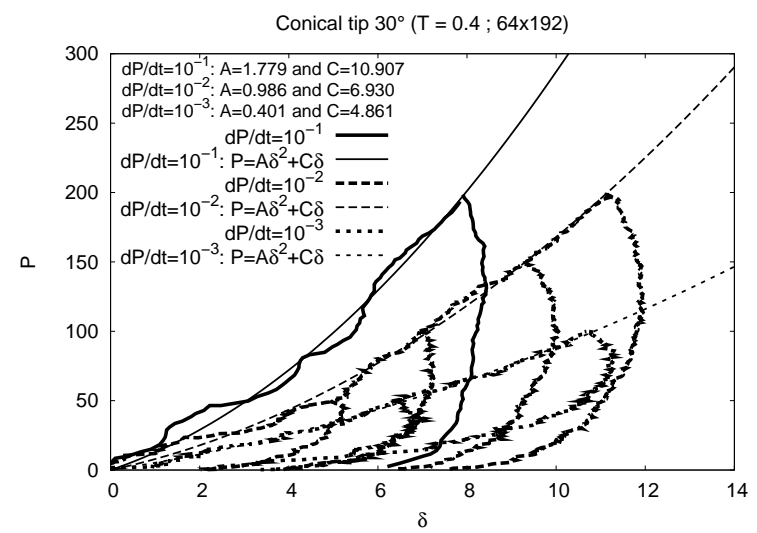

Figure 6. MD simulations of an indentation test with a linear $P$ function $(T=0.4)$ at various loading speeds denoted $d P / d t$. The regressions with their appropriate functions are shown for the loading phase.

Figure 6 depicts a test with a linear load $(P)$ loading function (several indentations at several loads). This figure shows that a velocity $(d P / d t)$ increase leads to an increase in the hardness of the polymer film at constant temperature. The parameter $A$ passes from 0.401 at $d P / d t=10^{-3}$ to 1.779 at $d P / d t=10^{-1}$, for a fixed temperature of $T=0.4$. This is a consequence of the polymer viscosity (viscoelasticity and/or viscoplasticity). The faster the indenter moves, the less chain rearrangement (i.e. "chain flowing", which dissipates a lot of energy) can occur after the perturbation and hence the stronger the required load on the tip. Other numerical data (not presented here) show the same results whatever the tip control (constant $d P / d t$ or $V$ ). Moreover, the dependence of the curve $(P$ vs $\delta)$ on the tip velocity seems to be weaker at $T=0.2$ (below the glass transition) than at $T=0.4$ (at the glass transition).

2.4.2. Study of the unloading During the unloading phase (as the indenter comes out of the polymer film), the curve $(P$ vs $\delta$ ) can give information about the stiffness of the film (i.e. the quantity $E_{\text {eq }}$ ). In the case of purely elastic unloading, which occurs in materials with elasto-plastic behavior or indentation tests with depth control, the work of Oliver et al. [4] shows that the unloading is well predicted by:

$$
P=A \cdot\left(\delta-\delta_{f}\right)^{m} .
$$

Moreover, the work of Oliver et al. gives a relation between the initial slope of the unloading curve $S=(d P / d \delta)_{\max }$ and the equivalent contact stiffness $E_{\text {eq }}$ [4]. Using the 
correction factor $\beta=1$ (instead of 1.09 , for a Poisson's ratio $\nu=0.4$ with equation (20) in [4]) and $\mathbf{A}_{\mathbf{c}}=\pi \tan ^{2} \theta \cdot \delta^{2}$ (apparent contact area), a rough estimation of $E_{\text {eq }}$ may be obtained as follows:

$$
E_{e q}=\frac{\sqrt{\pi} \cdot S}{2 \beta \cdot \sqrt{\mathbf{A}_{\mathbf{c}}}} \approx \frac{S}{2 \delta \cdot \tan \theta},
$$

where $S$ is calculated from equation (3),

$$
S=A m \cdot\left(\delta_{\max }-\delta_{f}\right)^{m-1} .
$$

From regressions of the unloading curves using equation (3) (for fixed $\delta_{f} ; A$ and $m$ are fitting-parameters), the initial slope of the unloading curve $(S)$ was computed from equation (5) for the polymer films $64 \times 192$ and $64 \times 1536$, as illustrated in figure 7 . The subscript $i$ indicates the number of the unloading curve at a specified confinement $\kappa$.

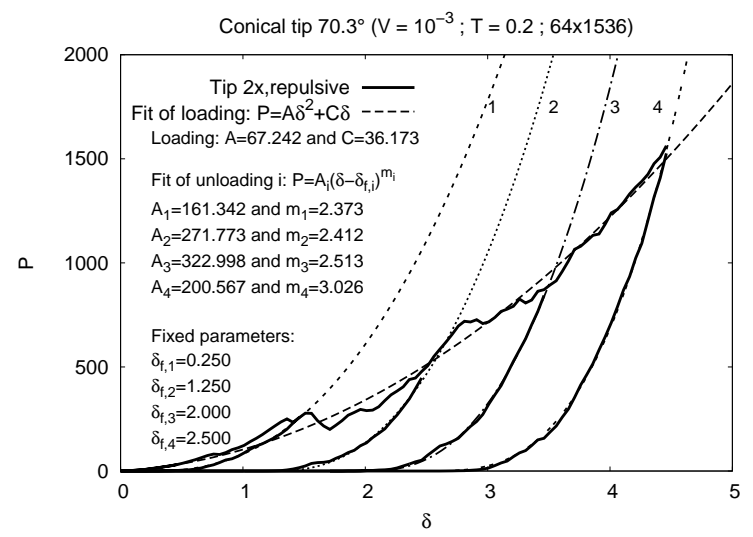

Figure 7. The slope $S$ is calculated at various confinements for the case of unloading with depth control. If the load is controlled, $S$ can be negative, due to creep.

Using figure 7 and equation (4), the equivalent contact stiffness $\left(E_{e q}=E /\left(1-\nu^{2}\right)\right.$ for indentation with a perfectly rigid indenter on a material of Young's modulus $E$ and Poisson's ratio $\nu$ ) is recapitulated as a function of the confinement in figure 8 . It is compared with results for the bulk obtained by uniaxial dynamic mechanical thermal analysis (DMTA) testing at a frequency of $10^{-3}$ on a cube of polymer $64 \times 192$. A first inspection of figure 8 shows good agreement between the values of $E_{e q}$ calculated from indentation tests and those derived from uniaxial DMTA tests. Nevertheless, the values of $E_{e q}$ are greater for the conical tip with $\theta=70.3^{\circ}$ than for the other tips. This may be attributed to a more important horizontal confinement, defined as $\kappa^{*}=2 a_{c} / \min \left(L_{x} ; L_{z}\right), \min \left(L_{x} ; L_{z}\right)$ denoting the smallest of the parmeters $L_{x}$ and $L_{z}$. For a vertical confinement of $\kappa=0.15$, one finds that $\kappa^{*}=0.39$ for $\theta=70.3^{\circ}, \kappa^{*}=0.26$ for $\theta=60^{\circ}, \kappa^{*}=0.15$ for $\theta=45^{\circ}$ and $\kappa^{*}=0.09$ for $\theta=30^{\circ}$, which justifies the above 
affirmation (in fact, $\min \left(L_{x} ; L_{z}\right)=30 \sigma$ for $\theta \in\left[30^{\circ} ; 60^{\circ}\right]$, and $\min \left(L_{x} ; L_{z}\right)=60 \sigma$ for $\left.\theta=70.3^{\circ}\right)$. Thus, for conical tip with $\theta=70.3^{\circ}$ in this specific situation, the indenter is more influenced by the periodic boundary conditions than for the other tips.

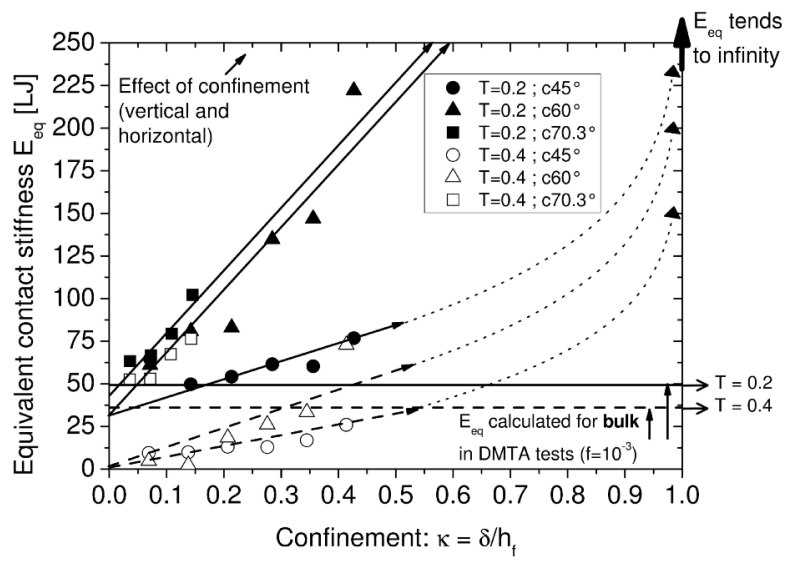

Figure 8. Equivalent contact stiffness calculated in indentation tests as a function of the vertical confinement $\kappa$. The results are compared with those for the bulk obtained in DMTA tests at a frequency of $10^{-3}$ on a cube of polymer $64 \times 192$.

\subsection{Recovery tests and film rheology}

Recovery tests were then simulated at $T=0.2\left(<T_{g}\right)$ and $T=0.4\left(\approx T_{g}\right)$ for a linear $\delta$ loading function $\left(V=d \delta / d t=10^{-3}\right)$ on $64 \times 192$ polymer films. The surface profiles after indentation for a conical tip with $\theta=45^{\circ}$ are presented in figures 9 and 10 .

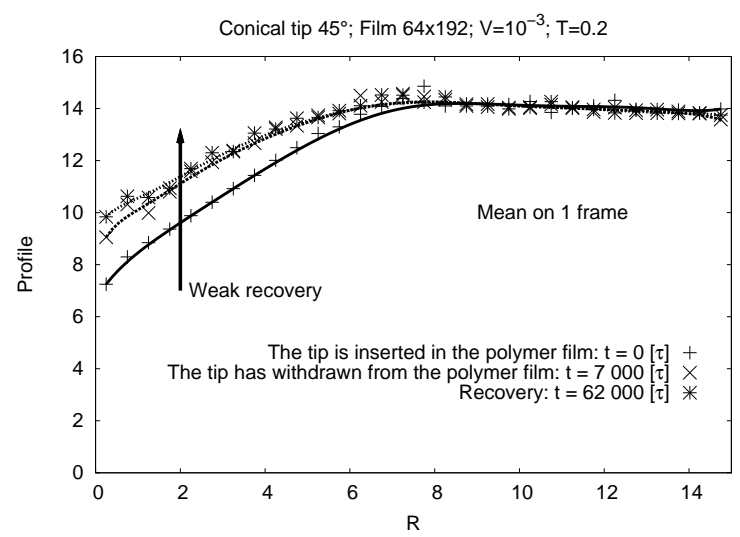

Figure 9. Recovery test at $T=0.2$. Radial surface profile at different times after indentation. Partial self healing can be observed (viscoplasticity dominates). 
To clearly depict the elastic or viscoelastic recovery, the surface profiles are only shown for one configuration, i.e. at one time $t \propto \tau$ (where $\tau$ is time in LJ units; see table 1). The tip withdraws progressively out of the polymer film over a period of $7000 \tau$ after an indentation test and recovery of the surface is observed from $t=7000 \tau$ to $t=62000 \tau$. Figure 9 (resp. figure 10) shows partial self healing at $T=0.2$ (resp. an almost complete self healing $T=0.4$ ).

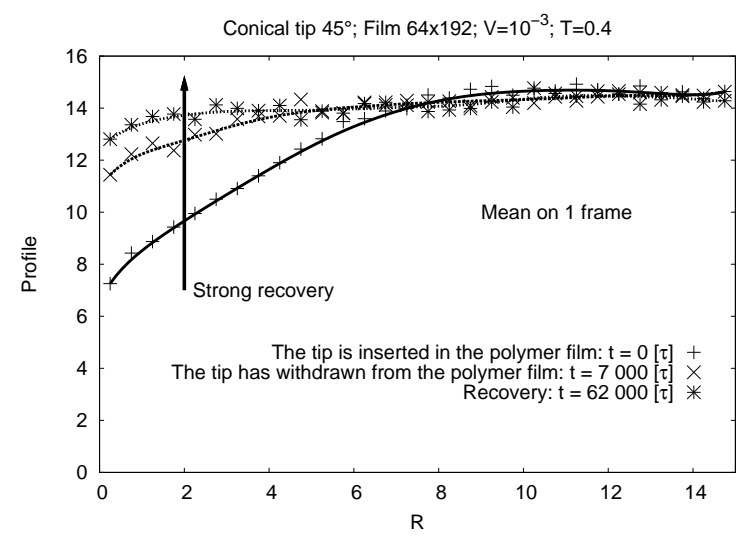

Figure 10. Recovery test at $T=0.4$. Radial surface profile at different times after indentation. Almost complete self healing can be observed (viscoelasticity dominates).

\section{Nano-indentation testing and experimental data}

Indentation tests are very convenient to investigate mechanical properties, despite some difficulties in the interpretation of the results due to the viscoelastic-plastic properties of polymeric materials (see for example [37]). Classical depth-sensing nano-indentation tests were performed at room temperature $\left(T_{r} \approx 22^{\circ} C\right)$ using a MTS Nano Indenter XP $\cap$. The indenter head was a MTS Nano DCM @ (Dynamic Contact Module), which allows highest resolution testing $(10[\mathrm{mN}]$ for a maximum load $P$, with a load resolution of $0.1[n N])$.

\subsection{Notation and experimental parameters}

The tip used was a sharp Berkovich diamond indenter, with an equivalent cone in conical approximation satisfying $\theta=70.3^{\circ}$. The equivalent tip defect (or tip radius $R_{\text {tip }}$ of the equivalent cone) was assumed to be small enough to ensure that $\delta_{\text {corr }}^{*} \approx 0.062 \cdot R_{\text {tip }}$ was negligible in comparison with $\delta$ (see figure 11), i.e. the tip was considered to be perfectly homothetic in the present study. In such cases, the representative strain is given by $\bar{\epsilon} \propto \cot \theta$ [38] and the representative strain rate by $\overline{\dot{\epsilon}} \propto \dot{P} / P$ [39]. During 
indentation on polymer surfaces with a linear loading function (which is widely used), the material is not solicited at a constant mean strain rate. An exponential loading function would be more appropriate [39], but this was not the subject of the present work. Hence the loading function was chosen to be linear (constant $d P / d t$ ), as in MD simulations. The loading and unloading speeds were equal. Tests were performed on two epoxy materials, denoted $E_{30}$ and $E_{50}$, which contain an epoxy resin (DER300 DOW Chemicals) crosslinked with agents marketed by DOW Chemicals (Jeffamine ED600, $T_{g} \approx 30^{\circ} \mathrm{C}$; Jeffamine $\mathrm{D} 400, T_{g} \approx 50^{\circ} \mathrm{C}$ ) in the course of a heating process (as described in the work of Charrault et al. [6]). The different lengths of these crosslinking agents allowed us to adjust the glass transitions of the two epoxy materials between $-30^{\circ} \mathrm{C}$ and $50^{\circ} \mathrm{C}$. Thus, the epoxy samples were characterized by DMTA testing, and the glass transitions were estimated to be $T_{g} \approx 30^{\circ} \mathrm{C}$ for $E_{30}\left(T_{g} \approx T_{r}\right)$ and $T_{g} \approx 50^{\circ} \mathrm{C}$ for $E_{50}$ $\left(T_{g} \gg T_{r}\right)$.

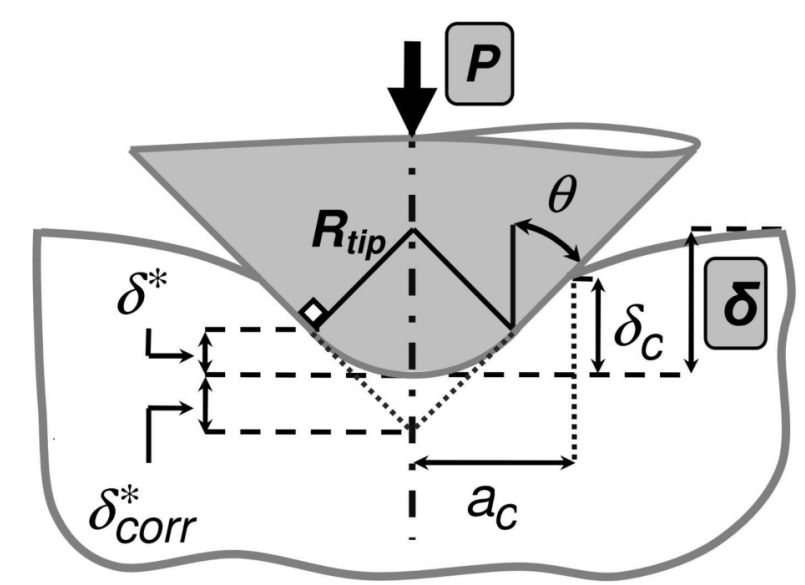

Figure 11. Notation used: $P$ is the load on the tip, $\delta$ the displacement under the surface and $a_{c}$ the contact radius.

\subsection{Nano-indentation tests on a semi-infinite medium}

It is well-known that a polymer behaves like an elastic-viscoplastic material at a temperature $T$ far from its glass transition $\left(T \ll T_{g}\right)$, and like a more or less viscoelastic rubber at temperatures near its glass transition $\left(T_{g} \approx T\right.$ or $\left.T_{g}<T\right)$. It is thus relevant to investigate the behavior of a polymer at different temperatures. However, nano-indentation tests with heating present as yet a technical challenge. Tests were therefore performed at room temperature on epoxy materials with different glass transitions. These tests were designed to provide a first point of comparison for MD simulations relating to the commonly studied load-depth $(P$ vs. $\delta$ ) curves (see figure 4$)$.

On inspection of the results, $E_{30}$ exhibited a priori elastic-viscoelastic-viscoplastic behavior with a strong dependence on $d P / d t$ (see figure 12). Moreover, it can be seen 
that the function $P=A \delta^{b}$ with $A>0$ and $b \leq 1$ fits the curve very well during the loading phase, where $A$ and $b$ are fitting-parameters accounting for the dependence on the mean strain rate $(d P / d t)$. The function $P=A \delta^{2}+C \delta$ is no longer valid in this case. During the unloading, strong "creep" was observed, reflected in the "nose" in the unloading curve. In contrast, $E_{50}$ exhibited a priori elastic-viscoelastic-viscoplastic behavior with a weak dependence on $d P / d t$ (see figure 12). The function $P=A \delta^{b}$ with $A>0$ and $b \geq 1$ fits the curve very well during the loading phase, as does the function $P=A \delta^{2}+C \delta$, which is a classical result for polymers [40]. During the unloading, no "creep" was observed for the loading functions investigated.

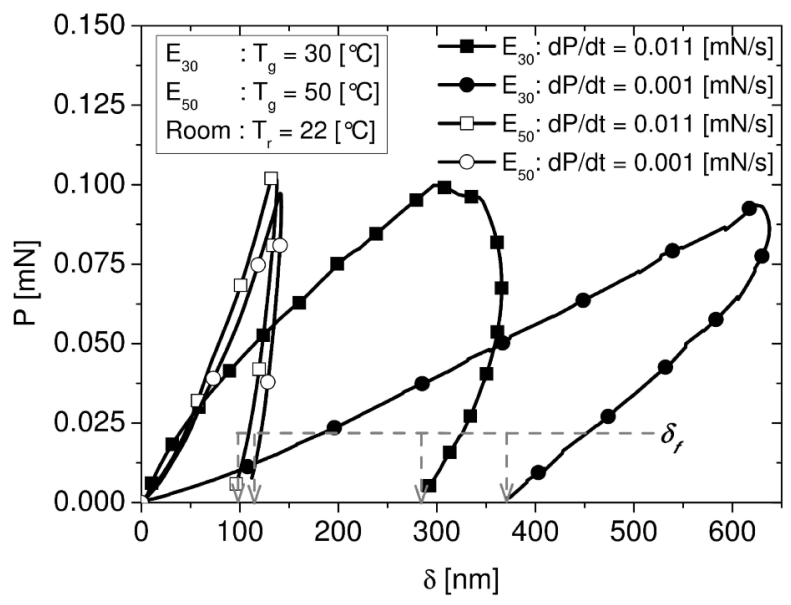

Figure 12. Nano-indentation tests on $E_{30}$ and $E_{50}$. The first (resp. the second) material exhibits a priori elastic-viscoelasticviscoplastic behavior with strong (resp. weak) dependence on $d P / d t$.

\subsection{Study of the recovery using AFM profilometry}

The indentation test was initially used to investigate the mechanical properties of materials with supposedly elasto-plastic behavior, i.e. with perfectly elastic unloading [4]. This assumption is convenient for tests on metals at room temperature or tests at a fast unloading speed on polymers, which exhibit in this case only low "creep" (due to viscoelasticity and / or viscoplasticity) during the unloading phase. Nevertheless, in our opinion, the nano-indentation test is not very appropriate to study the rheology of polymer surfaces near or above their glass transition, since this test does not allow one to observe the contact at all times. Firstly, the well-known "nose" during the unloading will alter the Young's modulus derived from the initial slope at the beginning of the unloading, since the unloading is no longer perfectly elastic. Various authors have tried to take into account the "creep" in the unloading (see for example [37]), but this phenomenon was not studied in the present work. Secondly, the observed residual depth at the end of the unloading (as the load $P$ approaches zero) may be attributed 
to viscoplasticity or viscoelasticity. It could be the consequence of a partial recovery which evolves with time. Consequently, AFM profilometry of $E_{30}$ and $E_{50}$ test samples was used to provide a better qualitative identification of the polymer rheology.

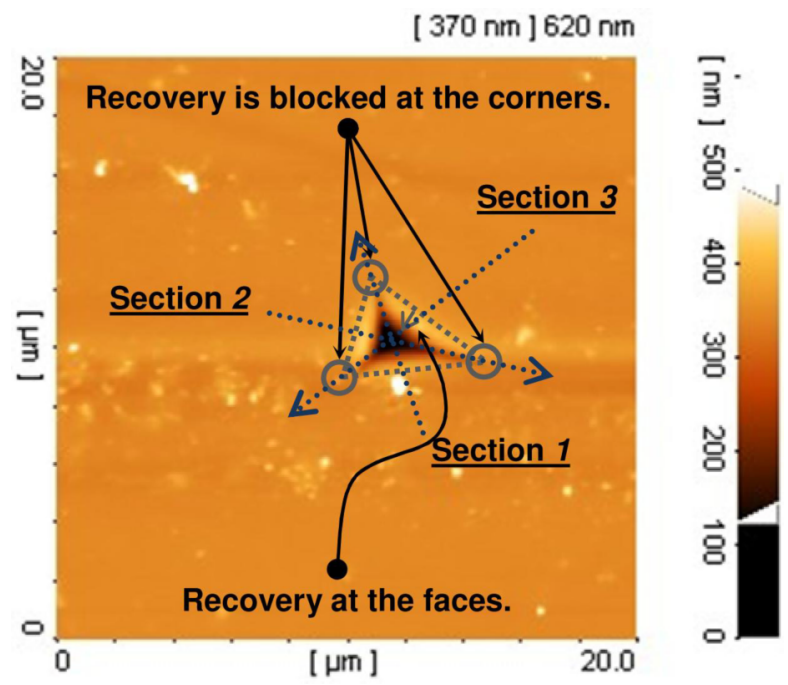

Figure 13. AFM profilometry $\approx 13[\mathrm{~min}]$ after indentation on $E_{50}$. The recovery is partial at the faces of the tip and blocked at the corners.

On epoxy $E_{50}$, a test at a maximal load of $P=1[\mathrm{mN}]$ showed a residual depth of $\approx 350[\mathrm{~nm}]$ at the end of the indentation. A first AFM profilometry was performed after $\approx 13[\mathrm{~min}]$, for technical reasons. The results are presented in figure 13 , and the corresponding profiles in figure 14 confirm a residual depth of $\approx 350[\mathrm{~nm}]$.

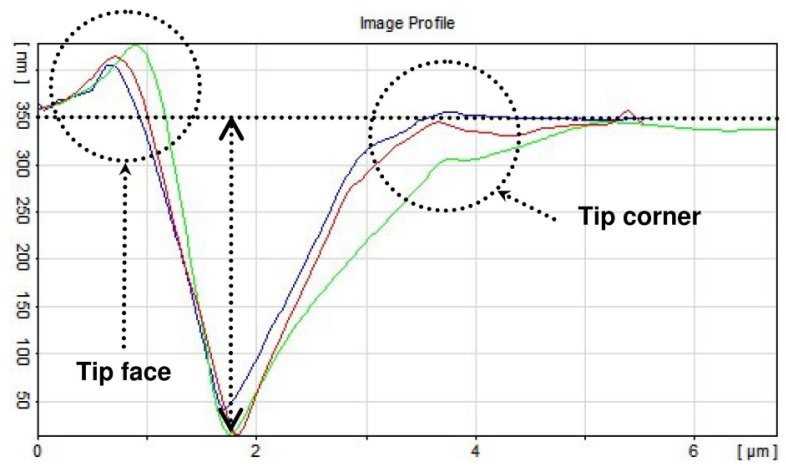

Figure 14. AFM profilometry $\approx 13[\mathrm{~min}]$ after indentation on $E_{50}$. Each profile represents a cross section of the imprint cut from one corner to the middle of the opposite face.

A second AFM profilometry was performed after $\approx 65[\mathrm{~min}]$. The profiles are presented in figure 15 and show a residual depth of $\approx 300[\mathrm{~nm}]$ : the recovery was very weak. This 
proved that $E_{50}$ displayed predominantly elastic-viscoplastic behavior, with a negligible viscoelastic contribution. Such behavior could indeed be expected from the nanoindentation tests presented in figure 12 . On epoxy $E_{30}$, a test at a maximal load of $P=1[m N]$ showed a residual depth at the end of the indentation resembling that figure 12. However, the AFM profilometry performed after $\approx 13[\mathrm{~min}]$ revealed no residual imprint. This demonstrated that there was complete recovery after $\approx 13[\mathrm{~min}]$ and that $E_{30}$ displayed a predominantly elastic-viscoelastic behavior, which could not be predicted from the tests in figure 12 .

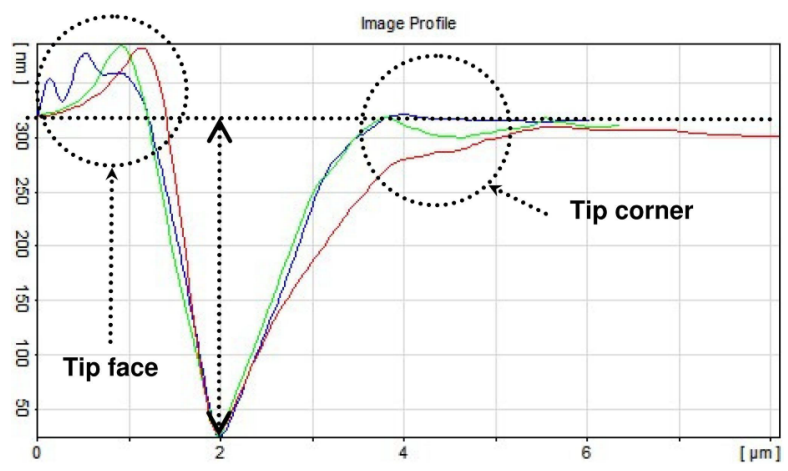

Figure 15. AFM profilometry $\approx 65[\mathrm{~min}]$ after indentation on $E_{50}$. Each profile represents a cross section of the imprint cut from one corner to the middle of the opposite face.

\section{Discussion}

The surface hardness $H$ of polymers (proportional to $P / \mathbf{A}_{\mathbf{c}}$ where $\mathbf{A}_{\mathbf{c}}$ is the apparent/projected contact area) is generally a function of the indenter geometry (spherical or conical), mechanical properties $\left(E, \sigma_{y}\right.$, etc $\left.\ldots\right)$ and a representative strain rate denoted $\dot{\epsilon}_{e q}$. Some studies of this function have been carried out for materials with elasto-plastic behavior (e.g. [41]). Nevertheless, such a function is not easy to establish analytically and experimentally for materials with time dependent behavior. It would be even more difficult to perform with MD simulations. Although it is possible to estimate mechanical properties with the aid of microscopic variables, for example using the fluctuation formalism for elastic constants (see [42, 43, 44, 45, 46]), this was not the subject of our paper. The present work was limited to a first qualitative analysis of the simulation results. Figure 16 shows the curves $\left(P / P_{\max }\right.$ vs $\left.\delta / \delta_{\max }\right)$ for the indentation tests on epoxy samples presented in figure 12 and for MD simulations of indentation tests on the modeled polymer films. There is good agreement between the experimental and numerical data, although the conditions controlling the load were not the same. These findings are promising for our future work. 


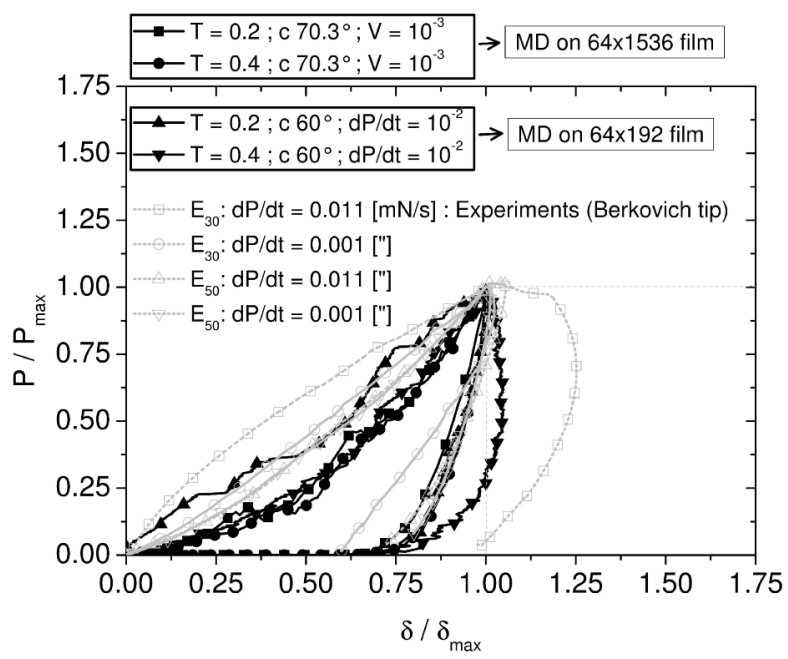

Figure 16. Comparison between experimental indentation tests on epoxy materials (figure 12) and numerical indentation tests performed by MD simulations. In this figure, the curves $\left(P / P_{\max }\right.$ vs $\left.\delta / \delta_{\max }\right)$ were computed at various speeds $V$ and $d P / d t$, so as to compare the experimental and numerical data.

\subsection{Indentation tests}

First of all, one can see that the MD simulations display good correlation with the experimental indentation data, with regard to the hysteresis of the $(P v s . \delta)$ curves and the polymer film rheology as a function of temperature (compare figures 5, 6 and 7 with figure 12). This is in spite of the fact that the simulated polymer film was not cross-linked but composed of linear chains, and that liquid-like behavior at $T \gg T_{g}$ would be expected for a linear chain model. Hence this first result is an interesting one which validates our contact modeling for MD simulations, although microscopic fluctuations for relatively small systems (here typically between $64 \cdot 192=12288$ and $64 \cdot 1536=98304$ particles, which is smaller than Avogadro's number $A$ with $A \approx 6.02 \cdot 10^{23}\left[\mathrm{~mol}^{-1}\right]$ ) can lead to difficulties in the interpretation of results. Secondly, figure 5 shows that no creep appears during the unloading and that MD simulations naturally predict a film hardness decreasing with temperature: the load $P$ is smaller at $T=0.4$ than at $T=0.2$ for the same depth $\delta$. Using FE simulations, such predictions would only be possible by implementing experimental laws of the type $E=f\left(T ; \dot{\epsilon}_{e q} ; t\right)$ or $\sigma_{y}=f\left(T ; \dot{\epsilon}_{e q} ; t\right)$. Turning to figure 6 , MD simulations predict that the film hardness will decrease with the loading speed at constant temperature: the depth $\delta$ is greater at $d P / d t=10^{-3}$ than at $d P / d t=10^{-1}$ for the same load $P$. However, creep occurs during the unloading (characterized by the "nose" in the unloading curves), particularly for $T=0.4$. This phenomenon is well known and, appears especially for a slow decay of $P$ and time dependent behavior. Lastly, as expected an influence of the perfectly 
rigid wall on the initial slope of the unloading curve $(P v s . \delta)$ is observed, as shown in figure 8. The deeper the tip penetrates into the film, the greater the initial slope $S$ and the stiffness $E_{e q}$, whereas $S$ and $E_{e q}$ should stay constant and be close to the bulk values in the case of indentation on a semi-infinite medium. The values of $E_{e q}$ given in figure 8 are in LJ units, where a value of 50 corresponds to $4.3[G P a]$ for PMMA [29]: these results are in good agreement with the experimental data. The values of $E_{e q}$ (obtained from indentation tests) are compared with those of the bulk (obtained from uniaxial DMTA tests at a frequency of $10^{-3}$ on a cube of polymer $\left.64 \times 192\right)$. These uniaxial DMTA test conditions are expected to be close to those of an indentation at $V=10^{-3}$ (with displacement control), although it is difficult to find a strict equivalence between a uniaxial and an indentation test. The results show good agreement between the two tests. At a temperature of $T=0.2$, where the visco-dependence of the polymer film behavior is weak, $E_{e q}$ tends to the value for the bulk with low confinement. At $T=0.4$, where the visco-dependence of the polymer film behavior is strong, $E_{e q}$ lies below the value for the bulk. This is probably due to the fact that the polymer film is at a temperature near its glass transition.

\subsection{Recovery tests}

Recovery tests likewise show that the material behavior is naturally predicted by MD simulations from thermodynamic parameters such as temperature. The surface profiles indicate that the polymer film passes progressively from predominantly viscoplastic behavior, where the recovery is partial over time (see figure 9), to predominantly viscoelastic behavior, where the recovery is quasi-total but delayed over time (see figure 10). The polymer film rheology thus shows good agreement with our experimental data for recovery tests. Conversely, FE simulations would require comparison with experiments and use of an elasto-viscoelasto-viscoplastic or similar law taking into account the dependence on $T$ and $\dot{\epsilon}_{e q}$. It is however important to note that it is not possible to define the polymer film rheology from the $(P v s . \delta)$ curve alone: a recovery test is also necessary.

\section{Conclusion and outlook}

In conclusion, the validation of a normal contact model using MD simulations was proposed in this paper. MD simulations give interesting results for contact mechanics and exhibit good correlation with experimental data. A strict comparison between MD simulation results (given in LJ units) and FE simulation results or experimental data (given in classical SI units) is difficult, because our study was restricted to a coarsegrained model. Nevertheless, MD and FE simulations can enrich one another: FE simulations can help in the conversion of LJ units, while MD simulations can enrich the models used in the FE formulation. In addition, MD simulations have the advantage of a microscopic thermodynamic formulation, which is more appropriate to study the 
local physics of a phenomenon. It was shown in the present work that MD simulations progressively adapt the polymer film rheology with temperature from $T<T_{g}$ to $T \approx T_{g}$ (recovery tests). Some objectives of our future work are to study the friction during scratch tests, the polymer chain orientation during normal and tangential contact and the stress under the indenter using the virial tensor, so as to evaluate the thickness $h$ of the small sheared layer described in the introduction. We will also attempt to establish a first link between MD and FE simulations in the context of contact mechanics.

\section{References}

[1] Gauthier C., Lafaye S., Schirrer R., Elastic recovery of a scratch in a polymeric surface: experiments and analysis, Tribology International (2001), 34, 469-479.

[2] Lafaye S., Gauthier C., Schirrer R., Analysis of the apparent friction of polymeric surfaces, J Mater Sci (2006), 41, 6441-6452.

[3] Pelletier H., Gauthier C., Schirrer R., Experimental and finite-element analysis of scratches on amorphous polymeric surfaces, Proc. IMechE Part J: J. Engineering Tribology (2008), 222.

[4] Oliver W.C., Pharr G.M., Measurement of hardness and elastic modulud by instrumented indentation: Advance in understanding and refinements to methodology, J. Mater. Res. 19 (1) (2004), 3-20.

[5] Cuenot S., Fretigny C., Demoustier-Champagne S., Nysten B. Measurement of elastic modulus of nanotubes by resonant contact atomic force microscopy, Journal of Applied Physics (2003), 93 (9), 5650-5655.

[6] Charrault E., Analyse mécanique et physico-chimique de la relation entre le frottement et l'adhésion, Ph.D. thesis in Polymer Physics. Strasbourg (France) : thesis UdS, 2007.

[7] Briscoe B. J., In: Friedrich K., Composite materials series - Volume 1: Friction and wear of polymer composites, Elsevier, Amsterdam (1986).

[8] Chen N., Maeda N., Tirrell M., Israelachvili J., Adhesion and Friction of Polymer Surfaces: The Effect of Chain Ends, Macromolecules (2005), 38, 3491-3503.

[9] Léger L., Hervet H., Bureau L., Friction mechanisms at polymer-solid interfaces, C. R. Chimie 9 (2006), 80-89.

[10] Lemaitre J., Chaboche J. L., Mechanics of Solid Materials, Dunod, Paris (1988), ISBN: 978 2100056620.

[11] Yashiro K., Itob T., Tomita Y. Molecular dynamics simulation of deformation behavior in amorphous polymer: nucleation of chain entanglements and network structure under uniaxial tension, International Journal of Mechanical Sciences (2003), 45, 1863-1876.

[12] Tsamados M., Tanguy A., Goldenberg C., Barrat J. L. Local elasticity map and plasticity in a model Lennard-Jones glass, Physical Review E (2009), 80, 026112, 1-17.

[13] Makke A., Perez M., Lame O., Barrat J. L. Mechanical testing of glassy and rubbery polymers in numerical simulations: Role of boundary conditions in tensile stress experiments, The Journal of Chemical Physics (2009), 131, 014904, 1-8.

[14] Badro J., Barrat J. L., Gillet P. Numerical Simulation of $\alpha$ - Quartz under Nonhydrostatic Compression: Memory Glass and Five-Coordinated Crystalline Phases, Physical Review Letters (1996), 76 (5), 772-775.

[15] Vladkov M., Barrat J. L. Linear and Nonlinear Viscoelasticity of a Model Unentangled Polymer Melt: Molecular Dynamics and Rouse Modes Analysis, Macromolecular Theory Simulations (2006), 15, 252-262.

[16] Szlufarska I., Chandross M., Carpick R. W., Recent advances in single-asperity nanotribology, Journal of Physics D: Applied Physics (2008), 41, 123001 (39pp). 
[17] Chandross M., Lorenz C. D., Stevens M. J., Grest G. S., Simulations of Nanotribology with Realistic Probe Tip Models, Langmuir (2008), 24, 1240-1246.

[18] Luan B. Q., Hyun S., Molinari J. F., Bernstein N., Robbins M. O., Multiscale modeling of twodimensional contacts, Physical Review E (2006), 74, 046710.

[19] Yang C., Persson B. N. J., Molecular Dynamics Study of Contact Mechanics: Contact Area and Interfacial Separation from Small to Full Contact, Physical Review Letters (2008), 100, 024303.

[20] Pätzold G., Linke A., Hapke T., Heermann D. W., Computer simulation of nano-indentation into polymer films, Thin Solids Films 295 (1997), 199-205.

[21] Pätzold G., Hapke T., Linke A., Heermann D. W., Deformation of polymer films by bending forces, Z. Phys. B 104 (1997), 513-521.

[22] Yashiro K., Furuta A., Tomita Y., Nanoindentation on crystal/amorphous polyethylene: Molecular dynamics study, Computational Materials Science 38 (2006), 136-143.

[23] Lee W.-J., Ju S.-P., Cheng C.-H., A Molecular Dynamics Study of Nanoindentation on a Methyl Methacrylate Ultrathin Film on a Au(111) Substrate: Interface and Thickness Effects, Langmuir (2008), 24, 13440-13449.

[24] Zhang L. C., Johnson K. L., Cheong W. C. D., A molecular dynamics study of scale effects on the friction of single-asperity contacts, Tribology Letters (2001), 10 (1-2), 23-28.

[25] Yiapanis G., Henry D. J., Evans E., Yarovsky I., Simulations of Nanoindentation of Polymer Surfaces: Effects of Surface Cross-Linking on Adhesion and Hardness, Journal of Physical Chemistry C (2010), 114, 478-486.

[26] Plimpton S., Fast parallel algorithms for short-range molecular dynamics, Journal of Computational Physics (1995), 117 (1), 1-19.

[27] Allen M. P., Tildesley D. J., Computer Simulation of Liquids, Oxford Science Publications, USA (1987), ISBN: 019855645.

[28] Frenkel D., Smit B., Understanding Molecular Simulation from Algorithms to Applications, Academic Press, UK (1996), ISBN: 0122673514.

[29] Schnell B., Etude par simulation numérique de la transition vitreuse et de l'état vitreux de polymères denses amorphes: propriétés mécaniques et phénomène de cavitation, Ph.D. thesis in Polymer Physics. Strasbourg (France) : thesis UdS, 2006.

[30] Everaers R., Sukumaran S. K., Grest G. S., Svaneborg C., Sivasubramanian A., Kremer K., Rheology and Microscopic Topology of Entangled Polymeric Liquids, Science (2004), Vol 303.

[31] Soddemann T., Dünweg B., Kremer K., Dissipative particle dynamics: A useful thermostat for equilibrium and nonequilibrium molecular dynamics simulations, Physical Review E 68 (2003), 046702-1 - 046702-8.

[32] Nose S., A unified formulation of the constant temperature molecular dynamics method, The Journal of Chemical Physics (1984), 81 (1), 511-519.

[33] Bennemann C., Paul W., Binder K., Dünweg B., Molecular-dynamics simulations of the thermal glass transition in polymermelts: $\alpha$-relaxation behavior, Physical Review E 57 (1998), 843-851.

[34] Peter S., Meyer H., Baschnagel J., Thickness-dependent reduction of the glass-transition temperature in thin polymer films with a free surface, Journal of Polymer Science B 44 (2006), 2951-2967.

[35] Peter S., Napolitano S., Meyer H., Wbbenhorst M., Baschnagel J., Modeling Dielectric Relaxation in Polymer Glass Simulations Dynamics in the Bulk and in Supported Polymer Films, Macromolecules (2008), 41, 7729-7743.

[36] Bucaille J. L., Simulation numérique de l'indentation et de la rayure des verres organiques, Ecole Nationale Supérieure des Mines de Paris, France, Ph.D. thesis in Materials Science, 2001.

[37] Ngan A. H. W., Wang H. T., Tang B., Sze K. Y., Correcting power-law viscoelastic effects in elastic modulus measurement using depth-sensing indentation, International Journal of Solids and Structures 42 (2005), 18311846.

[38] Bucaille J. L., Felder E., Hochstetter G., Identification of the viscoplastic behavior of a polycarbonate based on experiments and numerical modeling of the nano-indentation test, Journal 
of Materials Science 37 (2002), 3999-4011.

[39] Kermouche G., Loubet J. L., Bergheau J. M., Cone indentation of time-dependent materials: The effects of the indentation strain rate, Mechanics of Materials 39 (2007), 24-38.

[40] Cheng Y.-T., Cheng C.-M., Scaling, dimensional analysis, and indentation measurements, Materials Science and Engineering R 44 (2004), 91149

[41] Johnson K. L., Contact Mechanics, Cambridge University Press, UK (1985), ISBN-10: 0521347963.

[42] Papakonstantopoulos G. J., Doxastakis M., Nealey P. F., Barrat J.-L., de Pablo J. J., Calculation of local mechanical properties of filled polymers, Physical Review E 75 (2007), 031803.

[43] Farago O., Kantor Y., Fluctuation formalism for elastic constants in hard-spheres-and-tethers systems, Physical Review E (2000), Volume 61, Number 3.

[44] Lutsko J. F., Stress and elastic constants in anisotropic solids: Molecular dynamics techniques, J. App. Phys. (1988), 64:1152.

[45] Lutsko J. F., Generalized expressions for the calculation of elastic constants by computer simulation, J. App. Phys. (1989), 65:2991.

[46] Workum K. V., de Pablo J. J., Improved simulation method for the calculation of the elastic constants of crystalline and amorphous systems using strain fluctuations, Physical Review E 67 (2003), 011505. 